Mathematical Modelling And AnAlysis

Volume 19 Number 4, September 2014, 524-536 http://dx.doi.org/10.3846/13926292.2014.959084

(C) Vilnius Gediminas Technical University, 2014
Publisher: Taylor\&Francis and VGTU

http://www.tandfonline.com/TMMA

Print ISSN: 1392-6292

Online ISSN: 1648-3510

\title{
Global Existence Results for Functional Differential Inclusions with State-Dependent Delay
}

\section{Mouffak Benchohra ${ }^{a, c}$, Johnny Henderson ${ }^{b}$ and Imene Medjadj ${ }^{a}$}

${ }^{a}$ Laboratory of Mathematics, University of Sidi Bel-Abbès

P.O. Box 89, 22000 Sidi Bel-Abbès, Algeria

${ }^{b}$ Department of Mathematics, Baylor University

Waco, TX 76798-7328, USA

${ }^{c}$ Department of Mathematics, Faculty of Science, King Abdulaziz University

P.O. Box 80203, 21589 Jeddah, Saudi Arabia

E-mail(corresp.): benchohra@univ-sba.dz

E-mail: Johnny_Henderson@baylor.edu

E-mail: imene.medjadj@hotmail.fr

Received November 12, 2013; revised August 14, 2014; published online September 1, 2014

\begin{abstract}
Our aim in this work is to study the existence of solutions of a functional differential inclusion with state-dependent delay. We use the Bohnenblust-Karlin fixed point theorem for the existence of solutions.

Keywords: functional differential inclusion, mild solution, infinite and state-dependent delay, fixed point, semigroup theory.
\end{abstract}

AMS Subject Classification: 34G20; 34K20; 34K30.

\section{Introduction}

In this work we shall prove the existence of solutions of a functional differential inclusion. Our investigations will be situated in the Banach space of real functions which are defined, continuous and bounded on the real axis $(-\infty,+\infty)$. We will use Bohnenblust-Karlin's fixed theorem, combined with the Corduneanu's compactness criteria. More precisely we will consider the following problem:

$$
\begin{gathered}
y^{\prime}(t)-A y(t) \in F\left(t, y_{\rho\left(t, y_{t}\right)}\right), \quad \text { a.e. } t \in J:=[0,+\infty), \\
y(t)=\phi(t), \quad t \in(-\infty, 0],
\end{gathered}
$$

where $F: J \times \mathcal{B} \rightarrow \mathcal{P}(E)$ is a multivalued map with nonempty compact values, $\mathcal{P}(E)$ is the family of all nonempty subsets of $E, A: D(A) \subset E \rightarrow E$ is the 
infinitesimal generator of a strongly continuous semigroup $T(t), t \in J$, and $(E,|\cdot|)$ is a real Banach space. $\mathcal{B}$ is the phase space to be specified later, $\phi \in \mathcal{B}, \rho: J \times \mathcal{B} \rightarrow(-\infty,+\infty)$. For any function $y$ defined on $(-\infty,+\infty)$ and any $t \in J$ we denote by $y_{t}$ the element of $\mathcal{B}$ defined by

$$
y_{t}(\theta)=y(t+\theta), \quad \theta \in(-\infty, 0] .
$$

Here $y_{t}(\cdot)$ represents the history of the state from time $t-r$, up to the present time $t$. We assume that the histories $y_{t}$ to some abstract phases $\mathcal{B}$, to be specified later.

For modeling scientific phenomena where the delay is either a fixed constant or is given as an integral in which case is called distributed delay, we use differential delay equations or functional differential equations ; see for instance the books $[24,30,37]$.

An extensive theory is developed for evolution equations [2,3,21]. Uniqueness and existence results have been established recently for different evolution problems in the papers by Baghli and Benchohra for finite and infinite delay in $[6,7,8,9]$. However, complicated situations in which the delay depends on the unknown functions have been proposed in modeling in recent years. These equations are frequently called equations with state-dependent delay. Over the past several years it has become apparent that equations with state-dependent delay arise also in several areas such as in classical electrodynamics [20], in population models [10], in models of commodity price fluctuations [11,32], and in models of blood cell productions [33]. Existence results and among other things were derived recently for functional differential equations when the solution is depending on the delay on a bounded interval $[0, b]$ for impulsive problems. We refer the reader to the papers by Abada et al. [1], Ait Dads and Ezzinbi [16], Anguraj et al. [4], Hernandez et al. [27] and Li et al. [14,31]. See also $[5,12,25,26,35,36]$.

To the best of our knowledge, there exist very few papers devoted to functional evolution inclusions with state-dependent delay on unbounded intervals. Those results are stated in the Fréchet space setting. So the present results initiate the study of such problems in the Banach space setting.

\section{Preliminaries}

In this section we present briefly some notations and definition, and theorem which are used throughout this work.

In this paper, we will employ an axiomatic definition of the phase space $\mathcal{B}$ introduced by Hale and Kato in [23] and follow the terminology used in [28]. Thus, $\left(\mathcal{B},\|\cdot\|_{\mathcal{B}}\right)$ will be a seminormed linear space of functions mapping $(-\infty, 0]$ into $E$, and satisfying the following axioms:

$\left(A_{1}\right)$ If $y:(-\infty, b) \rightarrow E, b>0$, is continuous on $J$ and $y_{0} \in \mathcal{B}$, then for every $t \in J$ the following conditions hold:

(i) $y_{t} \in \mathcal{B}$;

(ii) There exists a positive constant $H$ such that $|y(t)| \leq H\left\|y_{t}\right\|_{\mathcal{B}}$; 
(iii) There exist two functions $L(\cdot), M(\cdot): \mathbb{R}^{+} \rightarrow \mathbb{R}^{+}$independent of $y$ with $L$ continuous and bounded, and $M$ bounded such that:

$$
\left\|y_{t}\right\|_{\mathcal{B}} \leq L(t) \sup \{|y(s)|: 0 \leq s \leq t\}+M(t)\left\|y_{0}\right\|_{\mathcal{B}}
$$

$\left(A_{2}\right)$ For the function $y$ in $\left(A_{1}\right), y_{t}$ is a $\mathcal{B}$-valued continuous function on $J$.

$\left(A_{3}\right)$ The space $\mathcal{B}$ is complete.

Denote

$$
L=\sup \{L(t): t \in J\}, \quad M=\sup \{M(t): t \in J\} .
$$

Remark 1. 1. (ii) is equivalent to $|\phi(0)| \leq H\|\phi\|_{\mathcal{B}}$ for every $\phi \in \mathcal{B}$.

2. Since $\|\cdot\|_{\mathcal{B}}$ is a seminorm, two elements $\phi, \psi \in \mathcal{B}$ can verify $\|\phi-\psi\|_{\mathcal{B}}=0$ without necessarily $\phi(\theta)=\psi(\theta)$ for all $\theta \leq 0$.

3. From the equivalence in the first remark, we can see that for all $\phi, \psi \in \mathcal{B}$ such that $\|\phi-\psi\|_{\mathcal{B}}=0$ : We necessarily have that $\phi(0)=\psi(0)$.

By $B U C$ we denote the space of bounded uniformly continuous functions defined from $(-\infty, 0]$ to $E$.

By $B C:=B C(-\infty,+\infty)$ we denote the Banach space of all bounded and continuous functions from $(-\infty,+\infty)$ into $E$ equipped with the standard norm

$$
\|y\|_{B C}=\sup _{t \in(-\infty,+\infty)}|y(t)| .
$$

Finally, by $B C^{\prime}:=B C^{\prime}([0,+\infty))$ we denote the Banach space of all bounded and continuous functions from $[0,+\infty)$ into $E$ equipped with the standard norm

$$
\|y\|_{B C^{\prime}}=\sup _{t \in[0,+\infty)}|y(t)| \text {. }
$$

Let $(E, d)$ be a metric space. We use the following notations:

$$
\mathcal{P}_{c l}(E)=\{Y \in \mathcal{P}(E): Y \text { closed }\}, \quad \mathcal{P}_{c v}(E)=\{Y \in \mathcal{P}(E): Y \text { convex }\} .
$$

Consider $H_{d}: \mathcal{P}(E) \times \mathcal{P}(E) \longrightarrow \mathbb{R}_{+} \cup\{\infty\}$, given by

$$
H_{d}(\mathcal{A}, \mathcal{B})=\max \left\{\sup _{a \in \mathcal{A}} d(a, \mathcal{B}), \sup _{b \in \mathcal{B}} d(\mathcal{A}, b)\right\}
$$

where $d(\mathcal{A}, b)=\inf _{a \in \mathcal{A}} d(a, b), d(a, \mathcal{B})=\inf _{b \in \mathcal{B}} d(a, b)$.

Definition 1. Let $X, Y$ be Hausdorff topological spaces and $F: X \rightarrow \mathcal{P}(Y)$ is called upper semi-continuous (u.s.c.) on $X$ if for each $x_{0} \in X$, the set $F(x)$ is a nonempty closed subset of $X$ and if for each open set $N$ of $X$ containing $F(x)$, there exists an open neighborhood $N_{0}$ of $x_{0}$ such that $F\left(N_{0}\right) \subseteq N$. 
Let $(E,\|\cdot\|)$ be a Banach space. A multivalued map $A: E \rightarrow \mathcal{P}(E)$ has convex (closed) values if $A(x)$ is convex (closed) for all $x \in E$. We say that $A$ is bounded on bounded sets if $A(B)$ is bounded in $E$ for each bounded set $B$ of $E$, i.e.,

$$
\sup _{x \in B}\{\sup \{\|y\|: y \in A(x)\}\}<\infty .
$$

$F$ is said to be completely continuous if $F(B)$ is relatively compact for every $B \in \mathcal{P}_{b}(E)$. If the multivalued map $F$ is completely continuous with non empty values, then $F$ is u.s.c. if and only if $F$ has a closed graph (i.e. $x_{n} \rightarrow x_{*}$, $y_{n} \rightarrow y_{*}, y_{n} \in F\left(x_{n}\right)$ implies $y_{*} \in F\left(x_{*}\right)$.

Definition 2. A function $F: J \times \mathcal{B} \longrightarrow \mathcal{P}(E)$ is said to be an $L^{1}$ - Carathéodory multivalued map if it satisfies:

(i) $y \mapsto F(t, y)$ is upper semicontinuous for almost all $t \in J$;

(ii) $t \mapsto F(t, y)$ is measurable for each $y \in \mathcal{B}$;

(iii) for every positive constant $l$ there exists $h_{l} \in L^{1}\left(J, \mathbb{R}^{+}\right)$

$$
\|F(t, y)\|=\sup \{|v|: v \in F(t, y)\} \leq h_{l}
$$

for all $|y| \leq l$ for almost all $t \in J$.

Lemma 1. Let $E$ be a Banach space. Let $F: J \times E \rightarrow \mathcal{P}_{c l, c v}(E)$ be a $L^{1}-$ Carathéodory multivalued map; and let $\Gamma$ be linear continuous from $L^{1}(J ; E)$ into $C(J ; E)$, then the operator

$$
\begin{aligned}
\Gamma \circ S_{F}: C(J, E) & \longrightarrow \mathcal{P}_{c p, c v}(C(J, X)), \\
y & \longmapsto\left(\Gamma \circ S_{F}\right)(y):=\Gamma\left(S_{F, y}\right)
\end{aligned}
$$

is a closed graph operator in $C(J ; X) \times C(J ; X)$.

Finally, we say that $A$ has a fixed point if there exists $x \in E$ such that $x \in A(x)$.

For each $y:(-\infty,+\infty) \rightarrow E$ let the set $S_{F, y}$ known as the set of selectors from $F$ defined by

$$
S_{F, y}=\left\{v \in L^{1}(J ; E): v(t) \in F\left(t, y_{\rho\left(t, y_{t}\right)}\right) \text {, a.e. } t \in J\right\} .
$$

For more details on multivalued maps we refer to the books of Deimling [17], Denkowski et al. [18,19], Górniewicz [22] and $\mathrm{Hu}$ and Papageorgiou [29].

Theorem 1 [Bohnenblust-Karlin fixed point [13]]. Let $B \in \mathcal{P}_{c l, c v}(E)$. And $N: B \rightarrow \mathcal{P}_{c l, c v}(B)$ be an upper semicontinuous operator and $N(B)$ is a relatively compact subset of $E$. Then $N$ has at least one fixed point in $B$.

Let us assume that $\Omega \neq \emptyset$ is a subset of $B C$, and let $N: \Omega \rightarrow \Omega$ and consider the solutions of the equation

$$
y(t) \in(N y)(t)
$$


Lemma 2 [Corduneanu [15]]. Let $D \subset B C([0,+\infty), E)$. Then $D$ is relatively compact if the following conditions hold:

(a) $D$ is bounded in $B C$.

(b) The function belonging to $D$ is almost equicontinuous on $[0,+\infty)$, i.e., equicontinuous on every compact subset of $[0,+\infty)$.

(c) The set $D(t):=\{y(t): y \in D\}$ is relatively compact subset on every compact of $[0,+\infty)$.

(d) The function from $D$ is equiconvergent, that is, given $\epsilon>0$, responds $T(\epsilon)>0$ such that $\left|u(t)-\lim _{t \rightarrow+\infty} u(t)\right|<\epsilon$, for any $t \geq T(\epsilon)$ and $u \in D$.

\section{Existence of Mild Solutions}

Now we give our main existence result for problem (1.1)-(1.2). Before stating and proving this result, we give the definition of the mild solution.

Definition 3. We say that a continuous function $y:(-\infty,+\infty) \rightarrow E$ is a mild solution of problem (1.1)-(1.2) if $y(t)=\phi(t)$ for all $t \in(-\infty, 0]$, and the restriction of $y(\cdot)$ to the interval $J$ is continuous and there exists $f(\cdot) \in$ $L^{1}(J ; E): f(t) \in F\left(t, y_{\rho\left(t, y_{t}\right)}\right)$ a.e. in $J$ such that $y$ satisfies the following integral equation

$$
y(t)=T(t) \phi(t)-\int_{0}^{t} T(t-s) f(s) d s \quad \text { for each } t \in J .
$$

Set

$$
\mathcal{R}\left(\rho^{-}\right)=\{\rho(s, \phi):(s, \phi) \in J \times \mathcal{B}, \rho(s, \phi) \leq 0\} .
$$

We always assume that $\rho: J \times \mathcal{B} \rightarrow \mathbb{R}$ is continuous. Additionally, we introduce the following hypothesis:

$\left(H_{\phi}\right)$ The function $t \rightarrow \phi_{t}$ is continuous from $\mathcal{R}\left(\rho^{-}\right)$into $\mathcal{B}$ and there exists a continuous and bounded function $\mathcal{L}^{\phi}: \mathcal{R}\left(\rho^{-}\right) \rightarrow(0, \infty)$ such that

$$
\left\|\phi_{t}\right\| \leq \mathcal{L}^{\phi}(t)\|\phi\| \quad \text { for every } t \in \mathcal{R}\left(\rho^{-}\right) .
$$

Remark 2. The condition $\left(H_{\phi}\right)$, is frequently verified by functions continuous and bounded. For more details, see for instance [28].

Lemma 3. [27, Lemma 2.4] If $y:(-\infty,+\infty) \rightarrow E$ is a function such that $y_{0}=\phi$, then

$$
\left\|y_{s}\right\|_{\mathcal{B}} \leq\left(M+\mathcal{L}^{\phi}\right)\|\phi\|_{\mathcal{B}}+L \sup \{|y(\theta)| ; \theta \in[0, \max \{0, s\}]\}, \quad s \in \mathcal{R}\left(\rho^{-}\right) \cup J,
$$

where $\mathcal{L}^{\phi}=\sup _{t \in \mathcal{R}\left(\rho^{-}\right)} \mathcal{L}^{\phi}(t)$. 
Let us introduce the following hypotheses:

$\left(H_{1}\right) \quad A: D(A) \subset E \rightarrow E$ is the infinitesimal generator of a strongly continuous semigroup $T(t), t \in J$ which is compact for $t>0$ in the Banach space $E$. Let $M^{\prime}=\sup \left\{\|T\|_{B(E)}: t \geq 0\right\}$.

$\left(H_{2}\right)$ The multifunction $F: J \times \mathcal{B} \longrightarrow \mathcal{P}(E)$ is Carathéodory with compact and convex values.

$\left(H_{3}\right)$ There exists a continuous function $k: J \rightarrow[0,+\infty)$ such that:

$$
H_{d}(F(t, u), F(t, v)) \leq k(t)\|u-v\|_{\mathcal{B}}
$$

for each $t \in J$ and for all $u, v \in \mathcal{B}$ and

$$
d(0, F(t, 0)) \leq k(t)
$$

with

$$
k^{*}:=\sup _{t \in J} \int_{0}^{t} k(s) d s<\infty .
$$

Theorem 2. Assume that $\left(H_{1}\right)-\left(H_{3}\right),\left(H_{\phi}\right)$ hold. If $k^{*} M^{\prime} L<1$, then the problem (1.1)-(1.2) has at least one mild solution on $B C$.

Proof. Transform the problem (1.1)-(1.2) into a fixed point problem. Consider the multivalued operator $N: B C \rightarrow \mathcal{P}(B C)$ defined by:

$$
N(y):=\left\{h \in B C: h(t)=\left\{\begin{array}{ll}
\phi(t), & \text { if } t \in(-\infty, 0] ; \\
T(t) \phi(0)+\int_{0}^{t} T(t-s) f(s) d s, & \text { if } t \in J,
\end{array}\right\}\right.
$$

where $f \in S_{F, y_{\rho\left(s, y_{s}\right)}}$.

Let $x(\cdot):(-\infty,+\infty) \rightarrow E$ be the function defined by:

$$
x(t)= \begin{cases}\phi(t), & \text { if } t \in(-\infty, 0] \\ T(t) \phi(0), & \text { if } t \in J\end{cases}
$$

Then $x_{0}=\phi$. For each $z \in B C$ with $z(0)=0$, we denote by $\bar{z}$ the function

$$
\bar{z}(t)= \begin{cases}0, & \text { if } t \in(-\infty, 0] \\ z(t), & \text { if } t \in J\end{cases}
$$

if $y(\cdot)$ satisfies (3.1), we can decompose it as $y(t)=z(t)+x(t), t \in J$, which implies $y_{t}=z_{t}+x_{t}$ for every $t \in J$ and the function $z(\cdot)$ satisfies

$$
z(t)=\int_{0}^{t} T(t-s) f(s) d s, \quad t \in J
$$

where $f \in S_{F, z_{\rho\left(s, z_{s}+x_{s}\right)}+x_{\rho\left(s, z_{s}+x_{s}\right)}}$. Set

$$
B C_{0}^{\prime}=\left\{z \in B C^{\prime}: z(0)=0\right\}
$$


and let

$$
\|z\|_{B C_{0}^{\prime}}=\sup \{|z(t)|: t \in J\}, \quad z \in B C_{0}^{\prime} .
$$

$B C_{0}^{\prime}$ is a Banach space with the norm $\|\cdot\|_{B C_{0}^{\prime}}$.

We define the operator $\mathcal{A}: B C_{0}^{\prime} \rightarrow \mathcal{P}\left(B C_{0}^{\prime}\right)$ by:

$$
\mathcal{A}(z):=\left\{h \in B C_{0}^{\prime}: h(t)=\left\{\begin{array}{ll}
0, & \text { if } t \leq 0 \\
\int_{0}^{t} T(t-s) f(s) d s, & \text { if } t \in J
\end{array}\right\}\right.
$$

where $f \in S_{F, z_{\rho\left(s, z_{s}+x_{s}\right)}+x_{\rho\left(s, z_{s}+x_{s}\right)}}$.

The operator $A$ maps $B C_{0}^{\prime}$ into $B C_{0}^{\prime}$, indeed the map $\mathcal{A}(z)$ is continuous on $[0,+\infty)$ for any $z \in B C_{0}^{\prime}, h \in \mathcal{A}(z)$ and for each $t \in J$ we have

$$
\begin{aligned}
|h(t)| & \leq M^{\prime} \int_{0}^{t}|f(s)| d s \\
& \leq M^{\prime} \int_{0}^{t}\left(k(s)\left\|z_{\rho\left(s, z_{s}+x_{s}\right)}+x_{\rho\left(s, z_{s}+x_{s}\right)}\right\|_{\mathcal{B}}+|F(s, 0)|\right) d s \\
& \leq M^{\prime} \int_{0}^{t} k(s) d s+M^{\prime} \int_{0}^{t} k(s)\left(L|z(s)|+\left(M+\mathcal{L}^{\phi}+L M^{\prime} H\right)\|\phi\|_{\mathcal{B}}\right) d s \\
& \leq M^{\prime} k^{*}+M^{\prime} \int_{0}^{t} k(s)\left(L|z(s)|+\left(M+\mathcal{L}^{\phi}+L M^{\prime} H\right)\|\phi\|_{\mathcal{B}}\right) d s .
\end{aligned}
$$

Set $C:=\left(M+\mathcal{L}^{\phi}+L M^{\prime} H\right)\|\phi\|_{\mathcal{B}}$. Then, we have

$$
\begin{aligned}
|h(t)| & \leq M^{\prime} k^{*}+M^{\prime} C \int_{0}^{t} k(s) d s+M^{\prime} \int_{0}^{t} L|z(s)| k(s) d s \\
& \leq M^{\prime} k^{*}+M^{\prime} C k^{*}+M^{\prime} L\|z\|_{B C_{0}^{\prime}} k^{*}
\end{aligned}
$$

Hence, $\mathcal{A}(z) \in B C_{0}^{\prime}$. Moreover, let $r>0$ be such that

$$
r \geq \frac{M^{\prime} k^{*}+M^{\prime} C k^{*}}{1-M^{\prime} k^{*} L}
$$

and $B_{r}$ be the closed ball in $B C_{0}^{\prime}$ centered at the origin and of radius $r$. Let $z \in B_{r}$ and $t \in[0,+\infty)$. Then

$$
|h(t)| \leq M^{\prime} k^{*}+M^{\prime} C k^{*}+M^{\prime} k^{*} L r .
$$

Thus $\|h\|_{B C_{0}^{\prime}} \leq r$, which means that the operator $\mathcal{A}$ transforms the ball $B_{r}$ into itself. Now we prove that $\mathcal{A}: B_{r} \rightarrow \mathcal{P}\left(B_{r}\right)$ satisfies the assumptions of Bohnenblust-Karlin's fixed theorem. The proof will be given in several steps.

Step 1: We shall show that the operator $\mathcal{A}$ is closed and convex. This will be given in several claims.

Claim 1. $\mathcal{A}(z)$ is closed for each $z \in B_{r}$. 
Let $\left(h_{n}\right)_{n \geq 0} \in \mathcal{A}(z)$ such that $h_{n} \rightarrow \tilde{h}$ in $B_{r}$. Then for $h_{n} \in B_{r}$ there exists $f_{n} \in S_{F, z_{\rho\left(s, z_{s}+x_{s}\right)}+x_{\rho\left(s, z_{s}+x_{s}\right)}}$ such that for each $t \in J$,

$$
h_{n}(t)=\int_{0}^{t} T(t-s) f_{n}(s) d s .
$$

Using the fact that $F$ has compact values and from hypotheses $\left(H_{2}\right),\left(H_{3}\right)$ we may pass a subsequence if necessary to get that $f_{n}$ converges to $f \in L^{1}(J, E)$

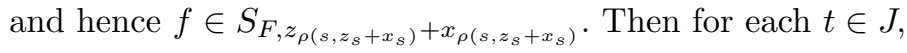

$$
h_{n}(t) \rightarrow \tilde{h}(t)=\int_{0}^{t} T(t-s) f(s) d s .
$$

So, $\tilde{h} \in \mathcal{A}(z)$.

Claim 2. $\mathcal{A}(z)$ is convex for each $z \in B_{r}$.

Let $h_{1}, h_{2} \in \mathcal{A}(z)$, then there exists $f_{1}, f_{2} \in S_{F, z_{\rho\left(s, z_{s}+x_{s}\right)}+x_{\rho\left(s, z_{s}+x_{s}\right)}}$ such that, for each $t \in J$ we have:

$$
h_{i}(t)=\int_{0}^{t} T(t-s) f_{i}(s) d s, \quad i=1,2 .
$$

Let $0 \leq \delta \leq 1$. Then, we have for each $t \in J$ :

$$
\left(\delta h_{1}+(1-\delta) h_{2}\right)(t)=\int_{0}^{t} T(t-s)\left[\delta f_{1}(s)+(1-\delta) f_{2}(s)\right] d s
$$

Since $F$ has convex values, one has $\delta h_{1}+(1-\delta) h_{2} \in \mathcal{A}(z)$.

Step 2: $\mathcal{A}\left(B_{r}\right) \subset B_{r}$ this is clear.

Step 3: $\mathcal{A}\left(B_{r}\right)$ is equicontinuous on every compact interval $[0, b]$ of $[0,+\infty)$ for $b>0$. Let $\tau_{1}, \tau_{2} \in[0, b], h \in \mathcal{A}(z)$ with $\tau_{2}>\tau_{1}$, we have:

$$
\begin{aligned}
& \left|h\left(\tau_{2}\right)-h\left(\tau_{1}\right)\right| \leq \int_{0}^{\tau_{1}}\left\|T\left(\tau_{2}-s\right)-T\left(\tau_{1}-s\right)\right\|_{B(E)}|f(s)| d s \\
& \quad+\int_{\tau_{1}}^{\tau_{2}}\left\|T\left(\tau_{2}-s\right)\right\|_{B(E)}|f(s)| d s \leq \int_{0}^{\tau_{1}}\left\|T\left(\tau_{2}-s\right)-T\left(\tau_{1}-s\right)\right\|_{B(E)} \\
& \quad \times\left(k(s)\left\|z_{\rho\left(s, z_{s}+x_{s}\right)}+x_{\rho\left(s, z_{s}+x_{s}\right)}\right\|_{\mathcal{B}}+|F(s, 0)|\right) d s \\
& \quad+\int_{\tau_{1}}^{\tau_{2}}\left\|T\left(\tau_{2}-s\right)\right\|_{B(E)}\left(k(s)\left\|z_{\rho\left(s, z_{s}+x_{s}\right)}+x_{\rho\left(s, z_{s}+x_{s}\right)}\right\|_{\mathcal{B}}+|F(s, 0)|\right) d s \\
& \leq C \int_{0}^{\tau_{1}}\left\|T\left(\tau_{2}-s\right)-T\left(\tau_{1}-s\right)\right\|_{B(E)} k(s) d s \\
& \quad+r L \int_{0}^{\tau_{1}}\left\|T\left(\tau_{2}-s\right)-T\left(\tau_{1}-s\right)\right\|_{B(E)} k(s) d s \\
& \quad+\int_{0}^{\tau_{1}}\left\|T\left(\tau_{2}-s\right)-T\left(\tau_{1}-s\right)\right\|_{B(E)} k(s) d s
\end{aligned}
$$




$$
\begin{aligned}
& +C \int_{\tau_{1}}^{\tau_{2}}\left\|T\left(\tau_{2}-s\right)\right\|_{B(E)} k(s) d s+r L \int_{\tau_{1}}^{\tau_{2}}\left\|T\left(\tau_{2}-s\right)\right\|_{B(E)} k(s) d s \\
& +\int_{\tau_{1}}^{\tau_{2}}\left\|T\left(\tau_{2}-s\right)\right\|_{B(E)} k(s) d s .
\end{aligned}
$$

When $\tau_{2} \rightarrow \tau_{2}$, the right-hand side of the above inequality tends to zero, since $T(t)$ is a strongly continuous operator and the compactness of $T(t)$ for $t>0$, implies the continuity in the uniform operator topology (see [34]), this proves the equicontinuity.

Step 4: $\mathcal{A}\left(B_{r}\right)$ is relatively compact on every compact interval of $[0, \infty)$.

Let $t \in[0, b]$ for $b>0$ and let $\varepsilon$ be a real number satisfying $0<\varepsilon<t$. For $z \in B_{r}$ we define

$$
h_{\varepsilon}(t)=T(\varepsilon) \int_{0}^{t-\varepsilon} T(t-s-\varepsilon) f(s) d s .
$$

Note that the set

$$
\left\{\int_{0}^{t-\varepsilon} T(t-s-\varepsilon) f(s) d s: z \in B_{r}\right\}
$$

is bounded:

$$
\left|\int_{0}^{t-\varepsilon} T(t-s-\varepsilon) f(s) d s\right| \leq r .
$$

Since $T(t)$ is a compact operator for $t>0$, the set, $\left\{h_{\varepsilon}(t): z \in B_{r}\right\}$ is precompact in $E$ for every $\varepsilon, 0<\varepsilon<t$. Moreover, for every $z \in B_{r}$ we have

$$
\begin{aligned}
\left|h(t)-h_{\varepsilon}(t)\right| & \leq M^{\prime} \int_{t-\varepsilon}^{t}|f(s)| d s \leq M^{\prime} \int_{t-\varepsilon}^{t} k(s) d s \\
& +M^{\prime} C \int_{t-\varepsilon}^{t} k(s) d s+r M^{\prime} \int_{t-\varepsilon}^{t} L k(s) d s \rightarrow 0 \quad \text { as } \varepsilon \rightarrow 0 .
\end{aligned}
$$

Therefore, the set $\left\{h(t): z \in B_{r}\right\}$ is precompact, i.e., relatively compact.

Step 5: $\mathcal{A}$ has closed graph.

Let $\left\{z_{n}\right\}$ be a sequence such that $z_{n} \rightarrow z_{*}, h_{n} \in \mathcal{A}\left(z_{n}\right)$ and $h_{n} \rightarrow h_{*}$. We shall show that $h_{*} \in \mathcal{A}\left(z_{*}\right)$. Note, that $h_{n} \in \mathcal{A}\left(z_{n}\right)$ means that there exists $f_{n} \in S_{F, z_{\rho\left(s, z_{s}^{n}+x_{s}\right)}^{n}}+x_{\rho\left(s, z_{s}^{n}+x_{s}\right)}$ such that

$$
h_{n}(t)=\int_{0}^{t} T(t-s) f_{n}(s) d s,
$$

we must prove that there exists $f_{*}$

$$
h_{*}(t)=\int_{0}^{t} T(t-s) f_{*}(s) d s .
$$


Consider the linear and continuous operator $K: L^{1}(J, E) \rightarrow B_{r}$ defined by

$$
K(v)(t)=\int_{0}^{t} T(t-s) v(s) d s
$$

we have

$$
\left|K\left(f_{n}\right)(t)-K\left(f_{*}\right)(t)\right|=\left|h_{n}(t)-h_{*}(t)\right| \leq\left\|h_{n}-h_{*}\right\|_{\infty} \rightarrow 0, \quad \text { as } n \rightarrow \infty .
$$

From Lemma 2.2 it follows that $K \circ S_{F}$ is a closed graph operator and from

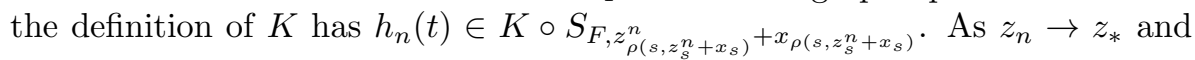
$h_{n} \rightarrow h_{*}$, there exists $f_{*} \in S_{F, z_{\rho\left(s, z_{s}^{*}+x_{s}\right)}^{*}+x_{\rho\left(s, z^{*}+x_{s}\right)}}$ such that:

$$
h_{*}(t)=\int_{0}^{t} T(t-s) f_{*}(s) d s .
$$

Hence the multivalued operator $\mathcal{A}$ is upper semi-continuous.

Step 6: $\mathcal{A}\left(B_{r}\right)$ is equiconvergent.

Let $z \in B_{r}$, we have, for $h \in \mathcal{A}(z)$ :

$$
\begin{aligned}
|h(t)| & \leq M^{\prime} \int_{0}^{t}|f(s)| d s \\
& \leq M^{\prime} k^{*}+M^{\prime} C \int_{0}^{t} k(s) d s+M^{\prime} r \int_{0}^{t} L k(s) d s \\
& \leq M^{\prime} k^{*}+M^{\prime} C \int_{0}^{t} k(s) d s+M^{\prime} r L \int_{0}^{t} k(s) d s .
\end{aligned}
$$

Then by (4), we have

$$
|h(t)| \rightarrow l \leq M^{\prime} k^{*}(1+C+r L), \quad \text { as } t \rightarrow+\infty .
$$

Hence,

$$
|h(t)-h(+\infty)| \rightarrow 0, \quad \text { as } t \rightarrow+\infty .
$$

As a consequence of Steps 1-6, with Lemma 2, we can conclude that $\mathcal{A}: B_{r} \rightarrow$ $\mathcal{P}\left(B_{r}\right)$ is continuous and compact. From Bohnenblust-Karlin's fixed theorem, we deduce that $\mathcal{A}$ has a fixed point $z^{*}$. Then $y^{*}=z^{*}+x$ is a fixed point of the operator $N$, which is a mild solution of the problem (1.1)-(1.2).

\section{An Example}

Consider the following functional partial differential equation

$$
\frac{\partial}{\partial t} z(t, x)-\frac{\partial^{2}}{\partial x^{2}} z(t, x) \in F(t, z(t-\sigma(t, z(t, 0)), x)),
$$


$x \in[0, \pi], t \in[0,+\infty)$,

$$
\begin{gathered}
z(t, 0)=z(t, \pi)=0, \quad t \in[0,+\infty), \\
z(\theta, x)=z_{0}(\theta, x), \quad t \in(-\infty, 0], x \in[0, \pi],
\end{gathered}
$$

where $F$ is a given multivalued map, and $\sigma:(-\infty,+\infty) \rightarrow \mathbb{R}^{+}$is continuous.

Take $E=L^{2}[0, \pi]$ and define $A: E \rightarrow E$ by $A \omega=\omega^{\prime \prime}$ with domain

$D(A)=\left\{\omega \in E, \omega, \omega^{\prime}\right.$ are absolutely continuous, $\left.\omega^{\prime \prime} \in E, \omega(0)=\omega(\pi)=0\right\}$.

Then

$$
A \omega=\sum_{n=1}^{\infty} n^{2}\left(\omega, \omega_{n}\right) \omega_{n}, \quad \omega \in D(A),
$$

where $\omega_{n}(s)=\sqrt{\frac{2}{\pi}} \sin n s, n=1,2, \ldots$ is the orthogonal set of eigenvectors of $A$. It is well known (see [34]) that $A$ is the infinitesimal generator of an analytic semigroup $T(t), t \geq 0$ in $E$ and is given by

$$
T(t) \omega=\sum_{n=1}^{\infty} \exp \left(-n^{2} t\right)\left(\omega, \omega_{n}\right) \omega_{n}, \quad \omega \in E .
$$

Since the analytic semigroup $T(t)$ is compact, there exists a positive constant $M$ such that

$$
\|T(t)\|_{B(E)} \leq M
$$

Let $\mathcal{B}=B C U\left(\mathcal{R}^{-} ; E\right)$ and $\phi \in \mathcal{B}$, then $\left(H_{\phi}\right)$, where $\rho(t, \varphi)=t-\sigma(\varphi)$.

Then the problem (1.1)-(1.2) in an abstract formulation of the problem $(4.1)-(4.3)$, and if the conditions $\left(H_{1}\right)-\left(H_{3}\right),\left(H_{\phi}\right)$ are satisfied, Theorem 2 implies that the problem (4.1)-(4.3) has at least one mild solutions on $B C$.

\section{Acknowledgement}

The authors are grateful to the referees for the careful reading of the paper and for their helpful remarks.

\section{References}

[1] N. Abada, R.P. Agarwal, M. Benchohra and H. Hammouche. Existence results for nondensely defined impulsive semilinear functional differential equations with state-dependent delay. Asian-Eur. J. Math., 2:449-468, 2008.

[2] N.U. Ahmed. Semigroup Theory with Applications to Systems and Control. Harlow John Wiley \& Sons, Inc., New York, 1991.

[3] N.U. Ahmed. Dynamic Systems and Control with Applications. World Scientific Publishing Co. Pte. Ltd., Hackensack, NJ, 2006.

[4] A. Anguraj, M.M. Arjunan and E.M. Hernàndez. Existence results for an impulsive neutral functional differential equation with state-dependent delay. Appl. Anal., 86:861-872, 2007. http://dx.doi.org/10.1080/00036810701354995. 
[5] D. Aoued and S. Baghli-Bendimerad. Mild solutions for perturbed evolution equations with infinite state-dependent delay. Electron. J. Qual. Theory Differ. Equ., 59:24 pp, 2013.

[6] S. Baghli and M. Benchohra. Uniqueness results for partial functional differential equations in Fréchet spaces. Fixed Point Theory, 9:395-406, 2008.

[7] S. Baghli and M. Benchohra. Existence results for semilinear neutral functional differential equations involving evolution operators in Fréchet spaces. Georgian Math. J., 17:423-436, 2010.

[8] S. Baghli and M. Benchohra. Global uniqueness results for partial functional and neutral functional evolution equations with infinite delay. Differential Integral Equations, 23:31-50, 2010.

[9] S. Baghli and M. Benchohra. Global uniqueness results for partial functional and neutral functional evolutions equations with state-dependent delay. Georgian Math. J., 2(3):35-52, 2010.

[10] J. Bélair. Population models with state-dependent delays. Lect. Notes Pure Appl. Math., 131:165-176, 1990.

[11] J. Bélair and M.C. Mackey. Consumer memory and price fluctuations on commodity markets: An integrodifferential model. J. Dynam. Differential Equations, 1:299-325, 1989. http://dx.doi.org/10.1007/BF01053930.

[12] M. Benchohra, I. Medjadj, J.J. Nieto and P. Prakash. Global existence for functional differential equations with state-dependent delay. J. Funct. Spaces Appl., p. 7, 2013. http://dx.doi.org/10.1155/2013/863561. Article ID 863561.

[13] H.F. Bohnenblust and S. Karlin. On a theorem of Ville. In A. Cañada, P. Drábek and A. Fonda(Eds.), Contribution to the Theory of Games, volume 24 of Ann. Math. Stud., pp. 155-160, Princeton, 1950. Princeton University Press.

[14] Y.-K. Chang, J.J. Nieto and Z.-H. Zhao. Existence results for a nondenselydefined impulsive neutral differential equation with state-dependent delay. Nonlinear Anal. Hybr. Syst., 4(3):593-599, 2010.

[15] C. Corduneanu. Integral Equations and Stability of Feedback Systems. Acedemic Press, New York, 1973.

[16] E. Ait Dads and K. Ezzinbi. Boundedness and almost periodicity for some state-dependent delay differential equations. Electron. J. Differential Equations, 2002(67):1-13, 2002.

[17] K. Deimling. Multivalued Differential Equations. Walter de Gruyter, Berlin, New York, 1992.

[18] Z. Denkowski, S. Migorski and N.S. Papageorgiou. An Introduction to Nonlinear Analysis: Applications. Kluwer Academic/Plenum Publishers, Boston, 2003.

[19] Z. Denkowski, S. Migorski and N.S. Papageorgiou. An Introduction to Nonlinear Analysis: Theory. Kluwer Academic/Plenum Publishers, Boston, 2003.

[20] R.D. Driver and M.J. Norris. Note on uniqueness for a one-dimensional two-body problem of classical electrodynamics. Ann. Phys., 42:347-351, 1967. http://dx.doi.org/10.1016/0003-4916(67)90076-0.

[21] A. Friedman. Partial Differential Equations. Holt, Rinehat and Winston, New York, 1969.

[22] L. Górniewicz. Topological Fixed Point Theory of Multivalued Mappings, volume 495 of Math. Appl. Kluwer Academic Publishers, Dordrecht, 1999. 
[23] J. Hale and J. Kato. Phase space for retarded equations with infinite delay. Funkcial. Ekvac., 21:11-41, 1978.

[24] J.K. Hale and S.M. Verduyn Lunel. Introduction to Functional Differential Equations, volume 99 of Appl. Math. Sci. Springer-Verlag, New York, 1993.

[25] F. Hartung. On second order differentiability with respect to parameters for differential equations with state-dependent delays. J. Dynam. Differ. Equ., 2013. http://dx.doi.org/10.1007/s10884-013-9330-5.

[26] F. Hartung. Parameter estimation by quasilinearization in differential equations with state-dependent delays. Discrete Contin. Dyn. Syst. Ser. B, 18:1611-1631, 2013. http://dx.doi.org/10.3934/dcdsb.2013.18.1611.

[27] E. Hernández, R. Sakthivel and A. Tanaka. Existence results for impulsive evolution differential equations with state-dependent delay. Electron. J. Differential Equations, 2008(28):1-11, 2008.

[28] Y. Hino, S. Murakami and T. Naito. Functional Differential Equations with Unbounded Delay. Springer-Verlag, Berlin, 1991.

[29] Sh. Hu and N. Papageorgiou. Handbook of Multivalued Analysis, Volume I: Theory. Kluwer, Dordrecht, Boston, London, 1997.

[30] V. Kolmanovskii and A. Myshkis. Introduction to the Theory and Applications of Functional-Differential Equations. Kluwer Academic Publishers, Dordrecht, 1999.

[31] W.S. Li, Y.K. Chang and J.J. Nieto. Solvability of impulsive neutral evolution differential inclusions with state- dependent delay. Math. Comput. Model., 49:1920-1927, 2009.

[32] M.C. Mackey. Commodity price fluctuations: price dependent delays and nonlinearities as explanatory factors. J. Econ. Theory, 48:497-509, 1989. http://dx.doi.org/10.1016/0022-0531(89)90039-2.

[33] M.C. Mackey and J. Milton. Feedback delays and the origin of blood cell dynamics. Comm. Theor. Biol., 1:299-327, 1990.

[34] A. Pazy. Semigroups of Linear Operators and Applications to Partial Differential Equations. Springer-Verlag, New York, 1983.

[35] A.V. Rezounenko. A condition on delay for differential equations with discrete state-dependent delay. J. Math. Anal. Appl., 385:506-516, 2012. http://dx.doi.org/10.1016/j.jmaa.2011.06.070.

[36] H.-O. Walther. A homoclinic loop generated by variable delay. J. Dynam. Differ. Equ., 2013. http://dx.doi.org/10.1007/s10884-013-9333-2.

[37] J. Wu. Theory and Applications of Partial Functional Differential Equations. Springer-Verlag, New York, 1996. 